\title{
Connecting the XYZ States at BESIII
}

\author{
Ryan E. Mitchell ${ }^{* \dagger}$ \\ Indiana University \\ E-mail: remitche@indiana.edu
}

\begin{abstract}
Most states in the charmonium system can be successfully described as a simple bound system composed of a charm quark and anti-charm quark. The past decade, however, has seen the discovery of a number of new states (named the " $X Y Z$ " states) that do not fit within this model, and which perhaps point towards more complex systems. In December of 2012, the BESIII Collaboration undertook a new program to study these $X Y Z$ states of charmonium by directly producing the $Y(4260)$ and $Y(4360)$ states in electron-positron collisions. Since that time, BESIII has collected the world's largest samples of $Y(4260)$ and $Y(4360)$ decays, which will help us understand those states, but which has also led to a number of surprises. Here I review several recent discoveries from these data samples.
\end{abstract}

52 International Winter Meeting on Nuclear Physics - Bormio 2014,

27-31 January 2014

Bormio, Italy

\footnotetext{
* Speaker.

${ }^{\dagger}$ This work was funded in part by a Chinese Academy of Sciences Young Scientist Fellowship.
} 


\section{Introduction: Directly Producing the Y(4260) and Y(4360) at BESIII}

The quark model of charmonium (for example, [1]) - in which a state of charmonium is described as a charm quark bound to an anticharm quark in a particular configuration of internal spin $(\mathbf{S})$, orbital angular momentum $(\mathbf{L})$, total spin $(\mathbf{J})$, and radial quantum numbers $(n)$ - has been remarkably successful in describing the spectrum of experimentally observed states below $D \bar{D}$ threshold. At higher masses, however, many new states have been discovered that seem to point beyond this simple $c \bar{c}$ picture [2]. These include candidates for quark-antiquark states with excited gluonic fields (hybrid mesons), four-quark states (tetraquarks), and meson-meson molecules. These " $X Y Z$ " states, if understood, could thus open the door to spectroscopy beyond the quark model.

Somewhat ironically, the majority of these new states were discovered by the Belle and BaBar experiments using $e^{+} e^{-}$collisions in the bottomonium region. BESIII, on the other hand, is in a unique position in that it can use $e^{+} e^{-}$collisions in the charmonium region, provided by the Beijing Electron Positron Collider (BEPC), to directly produce the $Y(4260)$ or $Y(4360)$ states by simply tuning the center of mass energies to 4.26 or $4.36 \mathrm{GeV}$, respectively. Between December of 2012 and June of 2013, BESIII collected a total of $2.9 \mathrm{fb}^{-1}$ of data in this energy region, with the largest samples being at $E_{C M}=4.23\left(1054 \mathrm{pb}^{-1}\right), 4.26\left(806 \mathrm{pb}^{-1}\right)$, and $4.36 \mathrm{GeV}\left(523 \mathrm{pb}^{-1}\right)$.

These energies have proved valuable not only for the production of the $Y(4260)$ and $Y(4360)$, but also for the production of "charged charmoniumlike states," the $Z_{c}(3900)$ and the $Z_{c}(4020)$, as well as radiative production of the $X(3872)$. The $Z_{c}(3900)$ and $Z_{c}(4020)$ are especially interesting because of their electric charge. Since a $c \bar{c}$ system is electrically neutral, these $Z_{c}$ states must contain more quarks, and may be four-quark systems, or molecules composed of two two-quark systems. Figure 1 shows the spectrum of charmonium and illustrates a few of the processes that have been observed by BESIII.

The results shown below establish the existence of the charged $Z_{c}(3900)$ and $Z_{c}(4020)$ structures. Using an angular analysis of its $D^{*} D$ decay the $Z_{c}(3900)$ is shown to have $J^{P}=1^{+}$. The $J^{P}$ of the $Z_{c}(4020)$ has not yet been determined. Amplitude analyses of the dominant reactions, especially $e^{+} e^{-} \rightarrow \pi^{+} \pi^{-} J / \psi$, are underway.

\section{Five XYZ Observations from BESIII in 2013}

\subsection{The Charged $\mathbf{Z}_{\mathbf{c}}(3900)$ in $\pi J / \psi$}

The $Y$ (4260) was originally discovered by BaBar [3], and later confirmed by Belle [4], in the Initial State Radiation (ISR) process $e^{+} e^{-}\left(\gamma_{I S R}\right) \rightarrow Y(4260) \rightarrow \pi^{+} \pi^{-} J / \psi$. BESIII confirmed the cross section for this process in early 2013 using $515 \mathrm{pb}^{-1}$ of data at $E_{C M}=4.26 \mathrm{GeV}$, near the peak of the $Y$ (4260) [5]. But more interestingly, BESIII also found structure in the $\pi^{ \pm} J / \psi$ subsystem. Fitting this structure, referred to as the $Z_{c}(3900)$, with a resonant lineshape (upper left of Fig. 2) resulted in a mass and width of $3899.0 \pm 3.6 \pm 4.9 \mathrm{MeV} / \mathrm{c}^{2}$ and $46 \pm 10 \pm 20 \mathrm{MeV}$, respectively. Since the $Z_{c}(3900)$ contains $c \bar{c}$, but is also charged, it must contain at least four quarks.

\subsection{Structure in Charged $D^{*} \overline{\mathbf{D}}+$ c.c.}

One possible clue for the interpretation of the $Z_{c}(3900)$ is that it has a mass very close to $D^{*} \bar{D}$ threshold. To investigate, BESIII analyzed the processes $e^{+} e^{-} \rightarrow \pi^{-} D^{+} \bar{D}^{* 0}+$ c.c. and $e^{+} e^{-} \rightarrow$ 


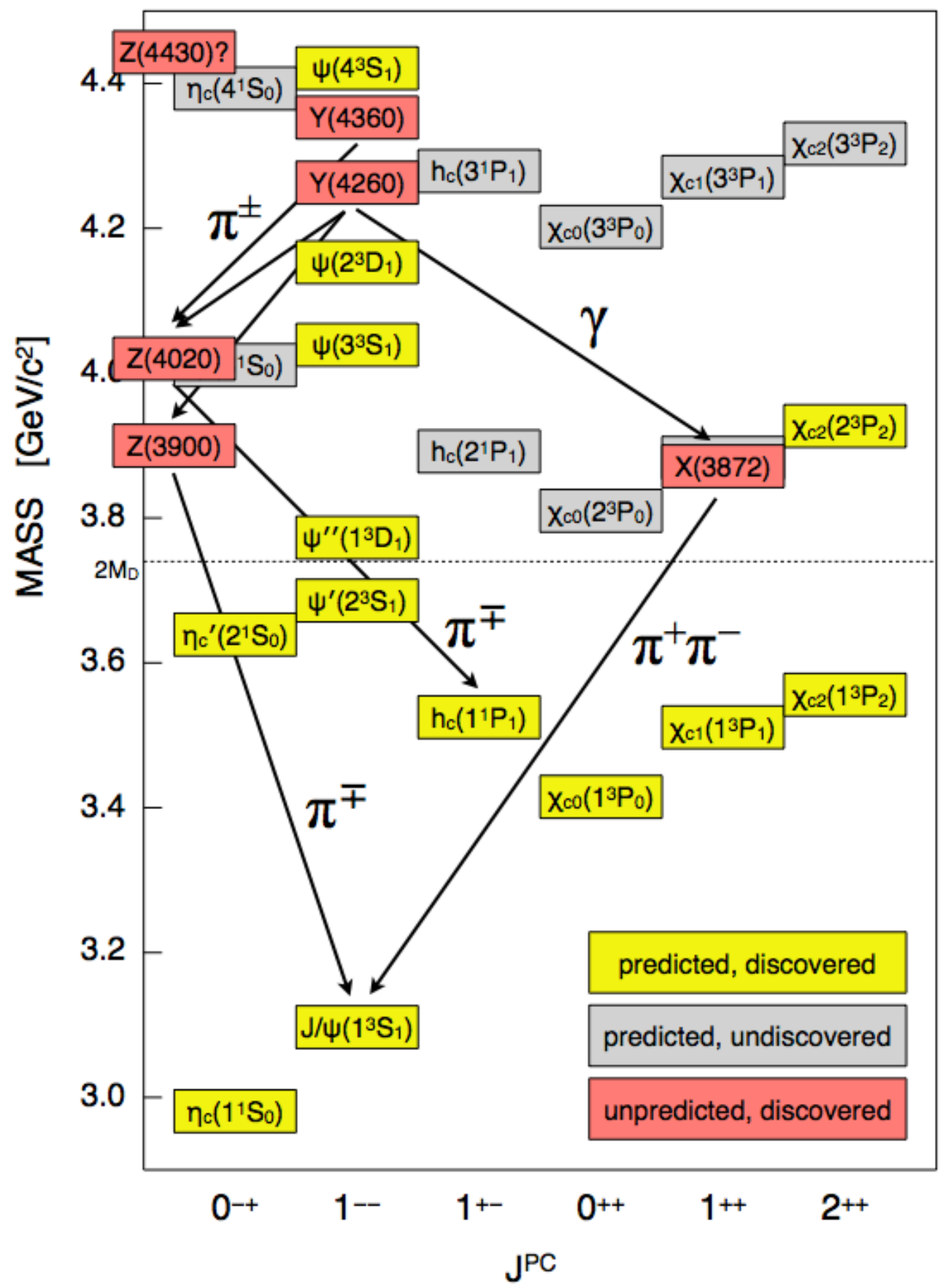

Figure 1: The charmonium system. The yellow and gray boxes represent states of charmonium as predicted by the quark model, where charmonium is treated as a bound system of a charm quark and anti-charm quark [1] - those in yellow have been experimentally discovered, while those in gray have not. The red boxes represent a sample of the so-called $X Y Z$ states of charmonium, which seem to point to configurations beyond the simple $c \bar{c}$ interpretation. The arrows mark transitions discussed in these proceedings. 
$\pi^{-} \bar{D}^{0} D^{*+}+$ c.c. at $E_{C M}=4.26 \mathrm{GeV}$ [6]. Clear structure in the mass spectrum of both $D^{+} \bar{D}^{* 0}+$ c.c. and $\bar{D}^{0} D^{*+}+$ c.c. was found (middle left of Fig. 2). The measured mass and width were $3883.9 \pm 1.5 \pm 4.2 \mathrm{MeV} / \mathrm{c}^{2}$ and $24.8 \pm 3.3 \pm 11.0 \mathrm{MeV}$, respectively. The mass and width are both slightly lower than those of the $Z_{c}(3900)$ in $\pi^{ \pm} J / \psi$, but it is likely the structures are related.

\subsection{The Charged $\mathrm{Z}_{\mathrm{c}}(\mathbf{4 0 2 0})$ in $\pi \mathrm{h}_{\mathrm{c}}$}

In previous work at CLEO-c, it was found that the process $e^{+} e^{-} \rightarrow \pi^{+} \pi^{-} h_{c}$ at $E_{C M}=4.17 \mathrm{GeV}$ was unexpectedly similar in strength to $e^{+} e^{-} \rightarrow \pi^{+} \pi^{-} J / \psi$ [7]. At BESIII, it was found that the process $e^{+} e^{-} \rightarrow \pi^{+} \pi^{-} h_{c}$ also remained strong at higher center of mass energies [8]. But more surprisingly, a narrow structure was found in the $\pi^{ \pm} h_{c}$ subsystem (upper right of Fig. 2). The mass and width of this structure, called the $Z_{c}(4020)$, were measured to be $4022.9 \pm 0.8 \pm 2.7 \mathrm{MeV} / \mathrm{c}^{2}$ and $7.9 \pm 2.7 \pm 2.6 \mathrm{MeV}$, respectively. Like the $Z_{c}(3900)$, the $Z_{c}(4020)$ is charged and so must contain more quarks than just the $c \bar{c}$.

\subsection{Structure in Charged $\mathbf{D}^{*} \overline{\mathbf{D}}^{*}$}

While the $Z_{c}(3900)$ is near $D^{*} \bar{D}$ threshold, the $Z_{c}(4020)$ is near $D^{*} \bar{D}^{*}$ threshold. Analyzing $e^{+} e^{-}$collisions at $4.26 \mathrm{GeV}$, BESIII found a structure decaying to $\left(D^{*} \bar{D}^{*}\right)^{ \pm}$in the process $e^{+} e^{-} \rightarrow$ $\pi^{\mp}\left(D^{*} \bar{D}^{*}\right)^{ \pm}$(middle right of Fig. 2) [9]. The mass and width of this structure, $4026.3 \pm 2.6 \pm$ 3.7 MeV/c ${ }^{2}$ and $24.8 \pm 5.6 \pm 7.7 \mathrm{MeV}$, respectively, are somewhat larger than those of the $Z_{c}(4020)$ in $\pi^{ \pm} h_{c}$, but close enough to suggest a connection.

\subsection{Observation of the $\mathrm{X}(\mathbf{3 8 7 2})$}

One final surprise from the 2013 data was the observation of the $X(3872)$ in the process $e^{+} e^{-} \rightarrow \gamma X$ (3872) with $X(3872) \rightarrow \pi^{+} \pi^{-} J / \psi$ (lower right of Fig. 2) [10]. This discovery presents a new way to access the $X(3872)$, which is still one of the least understood of the $X Y Z$ states. As briefly discussed below, the cross section of this process as a function of center of mass energy, while not conclusive, suggests this observation could arise from the process $Y(4260) \rightarrow \gamma X(3872)$. 

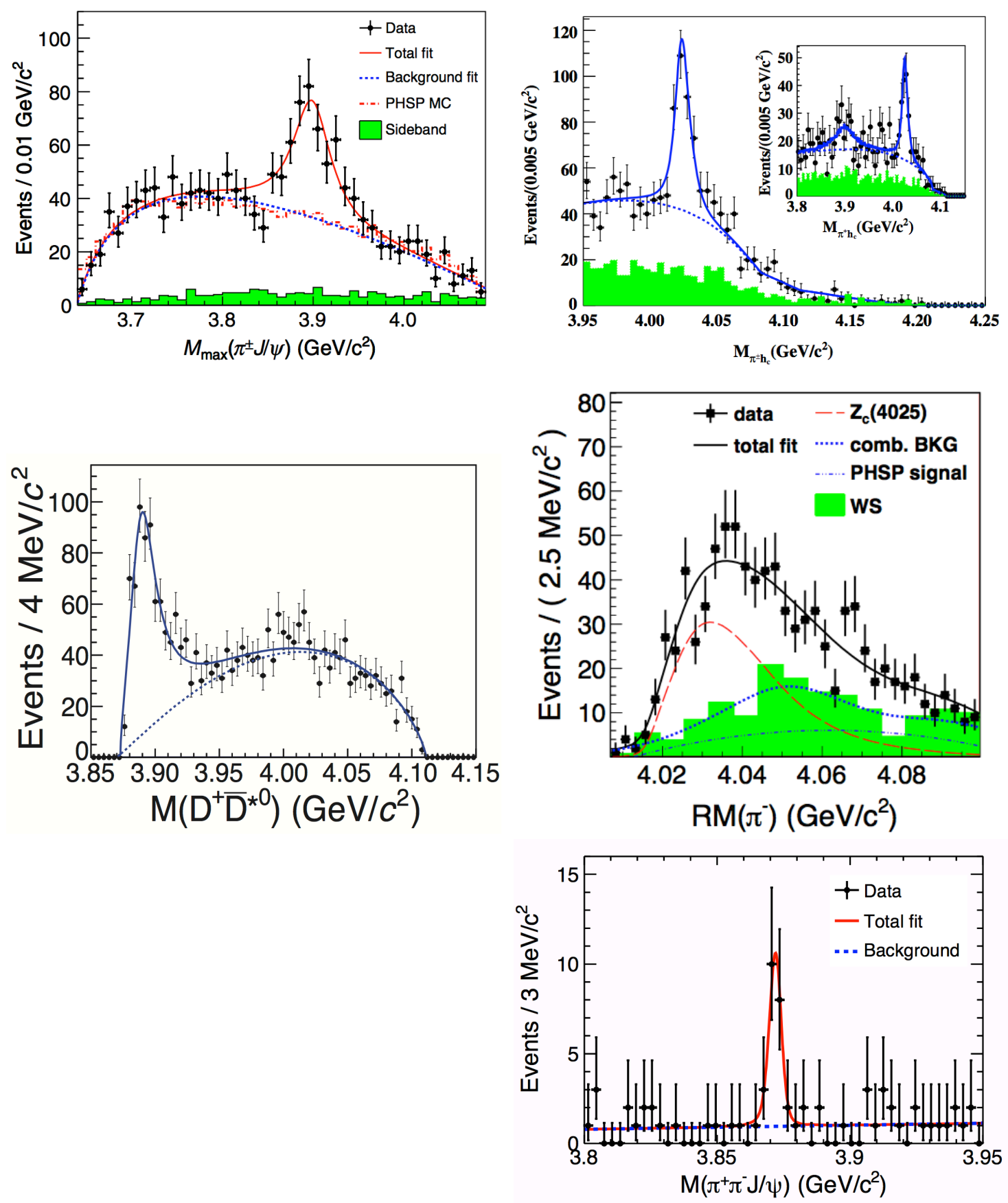

Figure 2: Five discoveries by the BESIII Experiment in 2013. (Upper Left) The $\mathbf{Z}_{\mathbf{c}}(\mathbf{3 9 0 0})$ produced in $e^{+} e^{-} \rightarrow \pi^{\mp} Z_{c}(3900)^{ \pm}$at $E_{C M}=4.26 \mathrm{GeV}$ and decaying through $Z_{c}(3900)^{ \pm} \rightarrow \pi^{ \pm} J / \psi$ [5]. (Upper Right) The $\mathbf{Z}_{\mathbf{c}}(\mathbf{4 0 2 0})$ produced in $e^{+} e^{-} \rightarrow \pi^{\mp} Z_{c}(4020)^{ \pm}$at $E_{C M}=4.23,4.26$, and $4.36 \mathrm{GeV}$ and decaying through $Z_{c}(4020)^{ \pm} \rightarrow \pi^{ \pm} h_{c}$ [8]. (Middle Left) Structure in $\mathbf{D}^{+} \overline{\mathbf{D}}^{* \mathbf{0}}+$ c.c. from the reaction $e^{+} e^{-} \rightarrow \pi^{-} D^{+} \bar{D}^{* 0}+$ c.c. at $E_{C M}=4.26 \mathrm{GeV}$ [6]. (Middle Right) Structure in $\left(\mathbf{D}^{*} \overline{\mathbf{D}}^{*}\right)^{ \pm}$from the reaction $e^{+} e^{-} \rightarrow \pi^{\mp}\left(D^{*} \bar{D}^{*}\right)^{ \pm}$at $E_{C M}=4.26 \mathrm{GeV}$ [9]. (Lower Right) The $\mathbf{X}(\mathbf{3 8 7 2})$ produced in $e^{+} e^{-} \rightarrow \gamma X(3872)$ at $E_{C M}=4.23,4.26$, and $4.36 \mathrm{GeV}$ and decaying through $X(3872) \rightarrow \pi^{+} \pi^{-} J / \psi[10]$. 


\section{Connections to the $Y(4260)$ and Outlook}

The fact that these five observations occur in $e^{+} e^{-}$collisions with center of mass energies near the mass of the $Y(4260)$ suggests that there may be an intimate connection between the $Y(4260)$ and the $Z_{c}(3900)$, the $Z_{c}(4020)$, and the $X(3872)$. Unfortunately, the BESIII data is not entirely conclusive in this regard. Figure 3 shows the cross sections of $e^{+} e^{-} \rightarrow \gamma X$ (3872) (top) and $e^{+} e^{-} \rightarrow$ $\pi^{+} \pi^{-} h_{c}$ (bottom) as a function of center of mass energy. For the former, the shape of the cross section is more consistent with the $Y(4260)$ than other shapes, but the significance is low. In the latter, the cross section shows more physics is present than just $Y(4260) \rightarrow \pi^{ \pm} Z_{c}(4020)^{\mp}$. A measurement of the production cross section of the $Z_{c}(3900)$ is still in progress.

\section{References}

[1] T. Barnes, S. Godfrey and E. S. Swanson, Phys. Rev. D 72, 054026 (2005).

[2] N. Brambilla, S. Eidelman, B. K. Heltsley, R. Vogt, G. T. Bodwin, E. Eichten, A. D. Frawley and A. B. Meyer et al., Eur. Phys. J. C 71, 1534 (2011).

[3] B. Aubert et al. [BaBar Collaboration], Phys. Rev. Lett. 95, 142001 (2005).

[4] C. Z. Yuan et al. [Belle Collaboration], Phys. Rev. Lett. 99, 182004 (2007).

[5] M. Ablikim et al. [BESIII Collaboration], Phys. Rev. Lett. 110, 252001 (2013).

[6] M. Ablikim et al. [BESIII Collaboration], Phys. Rev. Lett. 112, 022001 (2014).

[7] T. K. Pedlar et al. [CLEO Collaboration], Phys. Rev. Lett. 107, 041803 (2011).

[8] M. Ablikim et al. [BESIII Collaboration], Phys. Rev. Lett. 111, 242001 (2013).

[9] M. Ablikim et al. [BESIII Collaboration], Phys. Rev. Lett. 112, 132001 (2014).

[10] M. Ablikim et al. [BESIII Collaboration], Phys. Rev. Lett. 112, 092001 (2014). 

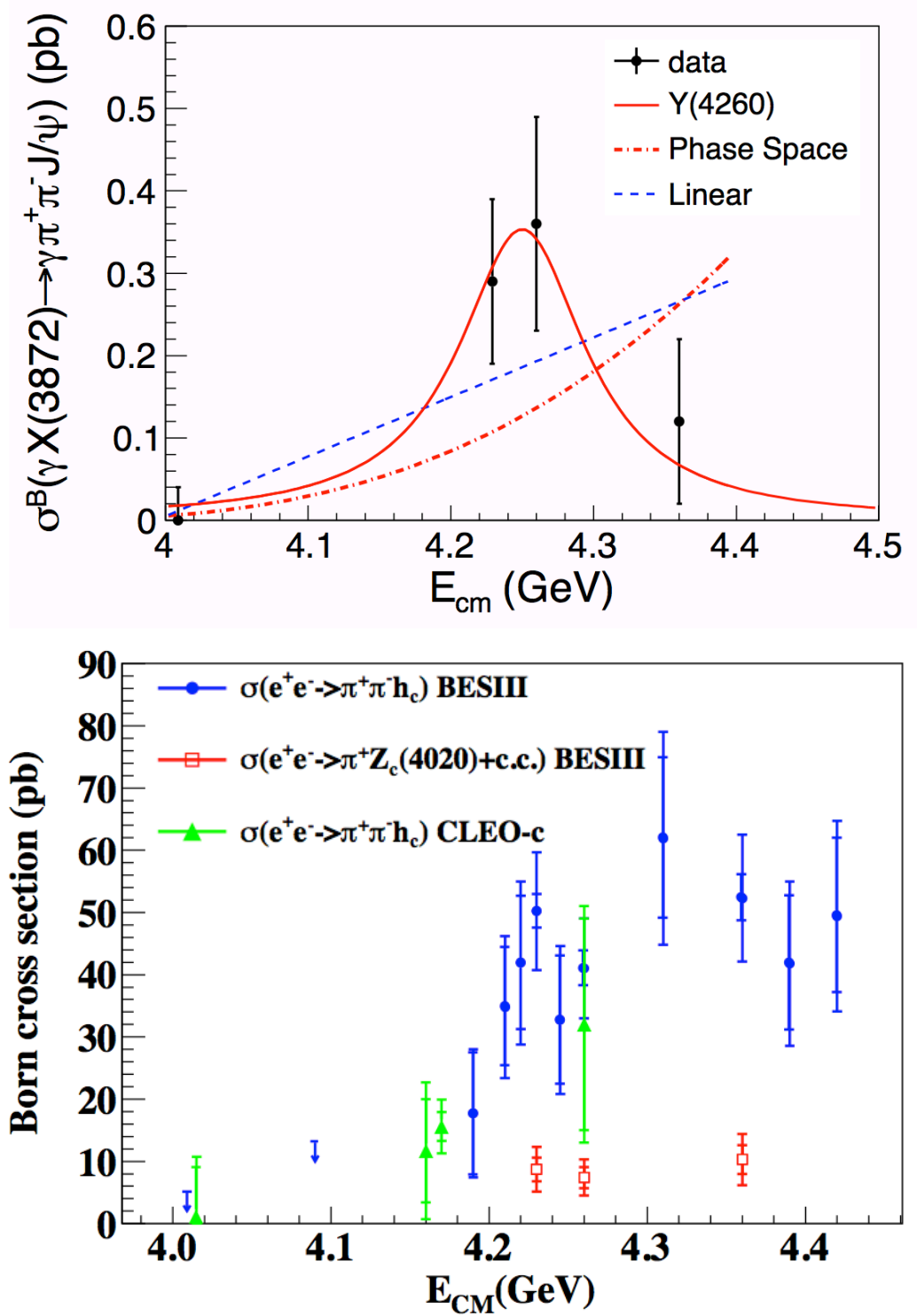

Figure 3: Connections to the $Y(4260)$. If a reaction originates from a $Y(4260)$ decay, the $e^{+} e^{-}$cross section as a function of center of mass energy should show structure consistent with the $Y(4260)$. (Top) The cross section for $e^{+} e^{-} \rightarrow \gamma X(3872)$. The cross section is more consistent with a $Y(4260)$ shape than phase space or a linear lineshape [10]. (Bottom) The cross sections for $e^{+} e^{-} \rightarrow \pi^{+} \pi^{-} h_{c}$ and $e^{+} e^{-} \rightarrow \pi^{ \pm} Z_{c}(4020)^{\mp}$. The shape of the cross section is inconclusive, but appears to stay constant at higher energies [8]. 